Published in final edited form as:

Curr Opin Cardiol. 2020 May ; 35(3): 276-281. doi:10.1097/HCO.0000000000000729.

\title{
Atrial arrhythmias in patients with left ventricular assist devices
}

\author{
Cevher Ozcan ${ }^{\mathrm{a}}$, Amrish Deshmukh ${ }^{\mathrm{b}}$ \\ aSection of Cardiology, Department of Medicine, Heart and Vascular Center, University of \\ Chicago Medical Center, Chicago, Illinois \\ bDivision of Cardiovascular Medicine, Department of Internal Medicine, Samuel and Jean Frankel \\ Cardiovascular Center, University of Michigan, Ann Arbor, Michigan, USA
}

\begin{abstract}
Purpose of review-Atrial arrhythmias commonly occur in patients with advanced heart failure with reduced ejection fraction (HFrEF) who require left ventricular assist devices (LVADs) implantation. This review summarizes the current literature regarding the incidence, prevalence, and predictors of atrial arrhythmias in LVAD patients and its impact on the clinical outcomes. Moreover, we review the mechanisms and management strategies of atrial arrhythmias in this population.

Recent findings-Atrial arrhythmias including atrial fibrillation, atrial flutter, and atrial tachycardia are highly prevalent in patients with advanced HFrEF before or after the LVAD implantation. Atrial arrhythmias have a significant impact on overall clinical outcome including survival, heart failure hospitalization, quality of life, thromboembolic events and resource utilization. Atrial fibrillation and other atrial arrhythmias frequently coexist in this population. In patients with atrial arrhythmias and LVAD, anticoagulation and cardiovascular implantable electronic devices should be closely monitored and managed to prevent thromboembolic events or inappropriate shocks. Rhythm and rate control strategies are comparable regarding overall clinical outcomes in this population. LVADs induce favorable atrial remodeling in patients with HFrEF.
\end{abstract}

Summary-Atrial arrhythmias are highly common in LVAD patients and have significant impact on overall clinical outcomes. Further studies are needed to determine optimal management and prevention of atrial arrhythmias in LVAD population.

\section{Keywords}

atrial arrhythmias; atrial fibrillation; heart failure; left ventricular assist devices

\section{INTRODUCTION}

Left ventricular assist devices (LVADs) are commonly used for patients with advanced heart failure with reduced ejection fraction (HFrEF), as both a bridge to transplantation or as

Correspondence to Cevher Ozcan, MD, Section of Cardiology, Department of Medicine, Heart and Vascular Center, University of Chicago Medical Center, 5841 S. Maryland Avenue, MC 6080, Chicago, IL 60637, USA. Tel: +1 773702 2559;

cozcan@uchicago.edu.

Conflicts of interest

There are no conflicts of interest. 
destination therapy $\left[1,2^{\mathbf{m}}\right]$. It is expected that number of patients who require LVAD will increase over time because of the increasing prevalence of HFrEF and the limited availability of transplant hearts. Understanding the relationship between LVADs and atrial arrhythmias is required to develop evidence-based strategies for the management of these patients. It is important to define the clinical characteristic, predictors, and treatment outcome in this population. Recent studies have shown that atrial arrhythmias, particularly atrial fibrillation, are highly prevalent in patients with advanced HFrEF before or after the LVAD implantation $\left[1,2 \mathbf{m}^{-}, 3-6,7,8,9\right]$. Atrial arrhythmias have significant clinical and therapeutic implications in this population. We review the literature on atrial arrhythmias in the LVAD population with a focus on epidemiology, predictors, outcomes, and management strategies including the rhythm versus rate control.

\section{EPIDEMIOLOGY AND PREDICTORS OF ATRIAL ARRHYTHMIAS IN THE LEFT VENTRICULAR ASSIST DEVICE POPULATION}

Atrial fibrillation and HFrEF commonly coexist and this combination is associated with an increased risk of all-cause mortality and morbidity relative to either condition in isolation $[1,2 \square, 3,4]$, Although specific data about other atrial arrhythmias are limited, the coexistence of atrial fibrillation and HFrEF causes a higher risk of stroke, hospitalization, dementia, kidney failure, myocardial infarction, and death than in those with heart failure or atrial fibrillation alone $[1,2-3,4]$, In addition to atrial fibrillation, the incidence and prevalence of other atrial arrhythmias including atrial flutter (AFL) and atrial tachycardia are high in patients with HFrEF with or without LVAD implantation [2 $\left[2^{\mathbf{a}}, 5,6\right]$, Atrial arrhythmias in HFrEF without LVAD are summarized elsewhere in this issue of the Journal. Here, we focus on atrial arrhythmias in patients with advanced HFrEF who require LVAD.

\section{Preleft ventricular assist devices prevalence of atrial arrhythmias}

Several investigators have demonstrated that atrial fibrillation is the most common sustained arrhythmia in pre-LVAD or post-LVAD patients with advanced $\mathrm{HFrEF}\left[5,6, \mathbf{\square}^{\mathbf{0}}, 8-10\right]$. A history of atrial fibrillation is present in $21-54 \%$ of patients with advanced HFrEF who are receiving LVADs $\left[2^{\boldsymbol{\square}}, 5,6,7^{\boldsymbol{\square}}, 8\right]$. Atrial fibrillation is found to be predominantly paroxysmal. Atrial tachycardia and AFL are reported in $15-22 \%$ of this population. AFL is the second most common atrial arrhythmia that experienced by $14 \%$ in pre-LVAD patients. The prevalence of atrial tachycardia is about $7 \%$ and atrioventricular nodal reentrant tachycardia $1 \%$. AFL and atrial tachycardia are often concomitantly existing with atrial fibrillation in these patients.

\section{Postleft ventricular assist devices incidence of atrial arrhythmias}

Patients with HFrEF who undergo LVAD implantation have an increased incidence of atrial arrhythmias, particularly atrial fibrillation $\left[2^{\boldsymbol{\square}}, 5,6,7^{\boldsymbol{\square}}, 8\right]$. The incidence of new onset atrial arrhythmias after LVAD is high in both the early postoperative period and afterward. Atrial fibrillation is documented in about $8 \%$ of patients after the first 30 days of LVAD [6,9,10]. Approximately 10-20\% of patients develop new onset atrial fibrillation in the year following LVAD implant $[5,6,7,8]$. In other cardiac surgery populations, postoperative atrial fibrillation occurs in 15-20\% of patients. Overall, incidence of de-novo post-LVAD atrial 
fibrillation is about $20-30 \%$ during long-term follow-up. Finally, in a significant minority of patients with atrial fibrillation prior to LVAD implantation (15-25\%), no atrial fibrillation detected in follow-up including on implantable device monitoring which may be due to favorable left atrial remodeling [5]. Atrial tachycardia is second most common new onset atrial arrhythmia after LVAD [6]. New onset AFL and atrioventricular nodal reentrant tachycardia are also documented [6]. In patients with paroxysmal atrial fibrillation, either de-novo or recurrent, about $9 \%$ progress to persistent atrial fibrillation after LVAD implantation in long-term follow-up [6].

\section{Predictors or atrial arrhythmias in left ventricular assist devices patients}

The risk of new onset atrial fibrillation in HFrEF without LVAD is higher with increasing age, decreased LV diastolic elastance, diabetes, hypertension, increased diuretic use, and renal impairment $\left[2^{-6}, 6\right]$. Similarly, older age, renal insufficiency, and lung disease are predictors of new onset atrial fibrillation after LVAD. In addition to those, ischemic cardiomyopathy, prior coronary artery bypass grafting, thyroid disease, and prolonged partial response interval (>200 ms) are associated with atrial fibrillation recurrence in postLVAD patients [6]. Male sex is more common in atrial fibrillation and AFL patients. But, there is no significant sex-based difference in overall clinical outcome.

\section{Pathophysiology of atrial arrhythmias in left ventricular assist devices patients}

The pathophysiology of atrial arrhythmias in HFrEF is complex and involves multiple interacting factors $\left[1,2 \mathbf{m}^{\mathbf{m}}, 6,7 \boldsymbol{\square}, 8-17\right]$. LVAD alters the relation between atrial arrhythmias and HFrEF by reducing left sided pressures with resulting left atrial remodeling $\left[5,6,7 \mathbf{\square}_{,-}\right.$ 16]. Atrial arrhythmias in HFrEF with or without LVAD is associated with complex atrial electroanatomical remodeling that is triggered by stretch, neurohormonal activation, and oxidative stress $\left[5,6,7 \mathbf{\square}_{, 8-16]}\right.$. Overall, LVADs induce favorable atrial structural and electrical remodeling by improving left atrial size and volume index. Unloading of the LV with LVAD generally reduces atrial pressures, valvular regurgitation, and atrial dimensions. This can reduce atrial fibrillation in long-term in some LVAD patients. Despite other improvements in left atrial parameters, preliminary evidence suggests that the left atrial appendage continues to have contractile dysfunction and may be a site of thrombosis despite mechanical unloading and anticoagulation with LVAD implantation [17-24]. Significant valvular disease can also persist in some patients and is associated with higher atrial pressures, atrial dilation, and early hospitalization and death [11,12]. However, no studies have directly correlated chronic valvular dysfunction or suboptimal hemodynamic assessments with incident atrial fibrillation or atrial arrhythmias in LVAD patients $[6,7]$. In the acute postoperative setting, atrial arrhythmias have been associated right ventricle (RV) failure suggesting that postoperative atrial arrhythmias may be due to elevated right atrial pressures [10]. Conversely, atrial fibrillation and atrial arrhythmias with rapid ventricular rates can cause RV failure [13-15]. At the molecular and cellular level, atrial fibrillation in HFrEF is associated with atrial energy deficit, oxidative, and metabolic stress [16]. This occurs with mitochondrial dysfunction [16]. Atrial tissue fibrosis, inflammation/infiltration, and cardiomyocyte loss have been shown as part of atrial remodeling with HFrEF, atrial arrhythmias, and LVAD. However, further mechanistic studies are warranted for understanding the molecular mechanism of the disease to develop preventive strategies. 
Impact of atrial arrhythmias on clinical outcomes in left ventricular assist devices patients

Atrial arrhythmias have significant effect on overall clinical outcomes in LVAD recipients

[2 ,5,15-27]. A preoperative history of persistent or permanent atrial fibrillation and atrial tachycardia has been associated with earlier heart failure hospitalization and mortality after LVAD placement $[5,6,15,20,21]$. This finding has not been uniformly replicated in all studies and paroxysmal atrial fibrillation without further classification have not been associated with increased mortality [ $\left.7^{\square}, 8,22-24\right]$. Though these series control for confounding patient factors, it is unclear whether persistent atrial fibrillation identifies patients with more comorbidities or whether persistent atrial arrhythmias independently impact outcomes. The latter possibility is suggested by evidence that atrial fibrillation is associated with right ventricular failure and ventricular arrhythmias after LVAD [10,15,2527]. LVAD patients with atrial fibrillation also have a higher risk of ventricular arrhythmia. Finally, the development of atrial fibrillation episodes after LVAD has also been associated with increased mortality $\left[2^{-}, 5,6\right]$.

In addition to mortality and hospitalization for heart failure, atrial fibrillation has been associated with other morbidity in LVAD patients including lower quality of life scores, 6min walk distance, and peak $\mathrm{VO}_{2}$ consumption $[5,14]$. As with other cardiac surgery populations, postoperative atrial fibrillation is associated with morbidity and resource utilization including prolonged mechanical ventilation, unanticipated right ventricular assist device use, discharge to facility, and prolonged ICU and hospital stay [10].

Although initial studies suggested that a history of atrial fibrillation was associated with increased risk of thromboembolism after LVAD, the majority of subsequent studies have demonstrated no increased risk of thromboembolic or bleeding events $\left[2 \mathbf{\square}_{, 5,6,7}, 8,9,17-\right.$ 20]. Though this data is heterogeneous with variable definitions of atrial fibrillation, differing anticoagulation and antiplatelet practices, and inclusion of different LVAD pump types, in totality it implies that the inherent thrombogenicity of LVAD therapy makes the contribution of atrial fibrillation to thromboembolic risk marginal. However, with new LVAD pump designs that carry a lower risk of hemocompatibility related events, atrial fibrillation does not appear to be associated with increased thromboembolic or bleeding outcomes [28].

\section{Management of atrial arrhythmias in left ventricular assist devices patients}

The management of atrial arrhythmias in LVAD patient is complex and similar to non-LVAD patient. There is insufficient data to change the current practice for particular therapeutic strategies in management of atrial arrhythmias in this population. In general, atrial arrhythmias in patients with LVAD are well tolerated. Yet, atrial arrhythmias may have significant hemodynamic impact in some LVAD patients. The management of these patients should be individualized regarding rhythm versus rate control or antiarrhythmic drug versus catheter ablation strategies for atrial arrhythmias $[1,2 \mathbf{m}, 3]$. Also, anticoagulation and cardiovascular implantable electronic devices (CIED) should be closely monitored and adjusted. 


\section{Rhythm versus rate control}

In retrospective series, there is no clear benefit or harm of pharmacologic rhythm control agents in LVAD patients with atrial fibrillation $\left[2 \mathbf{m}^{\mathbf{m}}\right]$. Rhythm control can be achieved by using class III antiarrhythmic drugs, amiodarone or dofetilide or sotalol, in this population [1,3]. Even though there is no reported negative impact of those agents on survival in patients with LVAD, antiarrhythmic agents should be administered with caution due to drug metabolism and potential side effects. Though rhythm control with catheter ablation has demonstrated morbidity and mortality benefit in patients with atrial fibrillation and heart failure without LVAD, similar data does not exist for patients with LVAD [29-31]. There is a paucity of data regarding catheter ablation of atrial fibrillation and other atrial arrhythmias in LVAD patients. In the largest series, eight patients with AFL, rapid ventricular response, and evidence of right ventricular failure underwent AFL ablation with improvement of right ventricle failure and resolution of inappropriate implantable cardioverter defibrillator shocks [13]. Outside of this report, two case reports describe ablation of AFL and pulmonary vein isolation for management of atrial arrhythmias causing rapid ventricular rates and decompensated heart failure [32,33]. Therefore, while ablation of AFL may be reasonable when rapid ventricular rates cause clear symptomatic or hemodynamic decompensation, there is no evidence that broader attempts at rhythm control therapy are beneficial. If atrial arrhythmias with rapid ventricular response compromise LVAD flows and performance, cardioversion should be considered to restore and maintain sinus rhythm in these patients. Rhythm control measures (antiarrhythmic drugs and electrical cardioversion) are not found to be associated with reduced mortality, thromboembolism, or bleeding in patients with atrial fibrillation.

\section{Medical management of rate control}

An effective for rate control strategy can be achieved by using $\beta$-blockers, with or without combination with digoxin. Target ventricular rate needs to be individualized based on patient's symptoms, hemodynamic status, and comorbidities. Also, digoxin is show to reduce the rate of heart failure-related hospitalizations in patients with HFrEF after LVAD implantation [34]. If $\beta$-blocker and digoxin combination is not sufficient to control ventricular rate, antiarrhythmic, particularly amiodarone can be considered as rate control agent in these patients. If atrial arrhythmias are drug-resistant or the patient is drugintolerant, it is reasonable to consider catheter ablation including atrioventricular nodal ablation [35].

\section{Cardiovascular implantable electronic devices and anticoagulation}

Majority of LVAD patients have CIED for the primary or secondary prevention of sudden cardiac death due to HFrEF or ventricular arrhythmias [1,2 $]$. In the MOMENTUM clinical trial, 68\% of HeartMate 3 recipients had CIED at the time of LVAD implantation [36]. Dynamic management of CIED after LVAD implantation is critical in patients with atrial arrhythmias to prevent inappropriate detection and therapies. Inappropriate shocks are common in LVAD patients with atrial arrhythmias, optimal and timely device programming must be done after LVAD implantation. Anticoagulation is critical in patients with atrial arrhythmias, and LVAD which requires close follow-up and management to diminish the 
risk of thromboembolic events and LVAD thrombus. However, left atrial appendage occlusion during LVAD implantation surgery decreases thromboembolic events and therefore, can be considered in patient who has pre-LVAD atrial fibrillation or AFL [18-20]. However, there are several areas that need to be investigated for better evidenced base management decisions. Those include subclinical incidental atrial fibrillation/AFL/atrial tachycardia detection on CIEDs, prevention of inappropriate shocks or thromboembolic events.

Regarding management of atrial arrhythmias in patients with LVAD, our recommendations based on current literature incorporated to recent consensus statement in Table 1 [ $\left.2^{\mathbf{m}}\right]$. Current studies are limited by their retrospective and single-center nature, and therefore, the variability in results is inevitable.

\section{CONCLUSION}

Based on the evidence to date, atrial arrhythmias are prevalent in LVAD patients and have significant impact in overall clinical outcome in this population. Despite current efforts for medical or catheter-based treatment, atrial arrhythmias remain an important determinant of morbidity and mortality in LVAD population in addition to the patients' underlying clinical characteristics. Thus, prospective multicenter studies are needed to explore impact of atrial arrhythmias in LVAD patient and also to determine optimal management strategy to improve clinical outcome in this population. Prevention of atrial arrhythmias in LVAD patients should be the ultimate goal.

\section{Financial support and sponsorship}

C.O. was supported by the National Institutes of Health/NHLBI (C.O., grant no. K08HL117082) for a related research project.

\section{REFERENCES AND RECOMMENDED READING}

Papers of particular interest, published within the annual period of review, have been highlighted as:

of special interest

$\square$ of outstanding interest

1. Yancy CW, Jessup M, Bozkurt B, et al. 2013 ACCF/AHA guideline for the management of heart failure: a report of the American College of Cardiology Foundation/American Heart Association Task Force on Practice Guidelines. J Am Coll Cardiol 2013; 62:e147-e239. [PubMed: 23747642]

2 . Gopinathannair R, Cornwell WK, Dukes JW, et al. Device therapy and arrhythmia management in left ventricular assist device recipients: a scientific statement from the American Heart Association. Circulation 2019; 139:e967-e989. [PubMed: 30943783] This consensus statement provides detail information about the management of various clinical conditions including atrial arrhythmias in the patient with left ventricular assist devises (LVADs).

3. January CT, Wann LS,Alpert JS, et al. 2014 AHA/ACC/HRS guideline for the management of patients with atrial fibrillation: executive summary: a report of the American College of Cardiology/ American Heart Association Task Force on practice guidelines and the Heart Rhythm Society. Circulation 2014; 130:2071-2104. [PubMed: 24682348] 
4. Benjamin EJ, Muntner P, Alonso A, et al. Heart disease and stroke statistics-2019 update: a report from the American Heart Association. Circulation 2019; 139:e56-e528. [PubMed: 30700139]

5. Enriquez AD, Calenda B, Gandhi PU, et al. Clinical impact of atrial fibrillation in patients with the HeartMate II left ventricular assist device. J Am Coll Cardiol 2014; 64:1883-1890. [PubMed: 25444141]

6. Deshmukh A, Kim G, Burke M, et al. Atrial arrhythmias and electroanatomical remodeling in patients with left ventricular assist devices. J Am Heart Assoc 2017; 6:1-9.

7 . Noll AE, Adewumi J, Amuthan R, et al. Atrial tachyarrhythmias among patients with left ventricular assist devices: prevalence, clinical outcomes, and impact of rhythm control strategies. JACC Clin Electrophysiol 2019; 5:459-466. [PubMed: 31000099] The study evaluated rhythm versus rate control strategy for atrial tachyarrhymias in patients with LVADs.

8. Hickey KT, Garan H, Mancini DM, et al. Atrial fibrillation in patients with left ventricular assist devices: incidence, predictors, and clinical outcomes. JACC Clin Electrophysiol 2016; 2:793-798. [PubMed: 29759763]

9. Deshmukh A, Bhatia A, Anyanwu E, et al. Incidence and outcomes of postoperative atrial fibrillation after left ventricular assist device. ASAIO J 2018; 64:581-585. [PubMed: 29485424]

10. Hawkins RB, Mehaffey JH, Guo A, et al. Postoperative atrial fibrillation is associated with increased morbidity and resource utilization after left ventricular assist device placement. J Thorac Cardiovasc Surg 2018; 156:1543-1549.e4. [PubMed: 29801690]

11. Tanaka A, Kitahara H, Onsager D, et al. Impact of residual valve disease on survival after implantation of left ventricular assist devices. Ann Thorac Surg 2018; 106:1789-1796. [PubMed: 30148976]

12. Kassis H, Cherukuri K, Agarwal R, et al. Significance of residual mitral regurgitation after continuous flow left ventricular assist device implantation. JACC Hear Fail 2017; 5:81-88.

13. Hottigoudar RU, Deam AG, Birks EJ, et al. Catheter ablation of atrial flutter in patients with left ventricular assist device improves symptoms of right heart failure. Congest Heart Fail 2013; 19:165-171. [PubMed: 23910701]

14. Brisco MA, Sundareswaran KS, Milano CA, et al. The incidence, risk, and consequences of atrial arrhythmias in patients with continuous-flow left ventricular assist devices. J Card Surg 2014; 29:572-580. [PubMed: 24750460]

15. Oezpeker C, Zittermann A, Pühler T, et al. Permanent atrial fibrillation and 2 year clinical outcomes in patients with a left ventricular assist device implant. ASAIO J 2017; 63:419-424. [PubMed: 28118262]

16. Ozcan C, Li Z, Kim G, et al. Molecular mechanism of the association between atrial fibrillation and heart failure includes energy metabolic dysregulation due to mitochondrial dysfunction. J Card Fail 2019; 25:911-920. [PubMed: 31415862]

17. Rahman A, Tam E, ZHeng L, et al. Peak left atrial appendage emptying velocity remains reduced in atrial fibrillation during continuous flow left ventricular assist device support. J Hear Lung Transplant 2019; 38:S45-S46.

18. Soufi MK, Roughneen PT, Al-Dossari GA, Hernandez-Montfort JA. Cerebrovascular accident in a patient with left ventricular assist device and therapeutic INR: should left atrial appendage be routinely closed? J Card Fail 2019; 25:

19. Lewis RS, Wang L, Spinelli KJ, et al. Surgical occlusion of the left atrial appendage and thromboembolic complications in patients with left ventricular assist devices. J Hear Lung Transplant 2017; 36:586-588.

20. Deshmukh A, Bhatia A, Sayer GT, et al. Left atrial appendage occlusion with left ventricular assist device decreases thromboembolic events. Ann Thorac Surg 2019; 107:1181-1186. [PubMed: 30365959]

21. Enciso JS, Seo J, Greenberg B, et al. Impact of age and left atrial remodeling in patients with atrial fibrillation undergoing left ventricular assist device implantation. J Card Fail 2019; 25:S40.

22. Pedde D, Soltani S, Stein J, et al. Impact of preoperative atrial fibrillation on thromboembolic events and pump thrombosis in long-term left ventricular assist device therapy. Eur J Cardiothoracic Surg 2020; 57:325-330. 
23. Xia Y, Stern D, Friedmann P, Goldstein D. Preoperative atrial fibrillation may not increase thromboembolic events in left ventricular assist device recipients on midterm follow-up. J Hear Lung Transplant 2016; 35:906-912.

24. Xuereb L, Go PH, Kaur B, et al. Impact of preoperative atrial fibrillation on postoperative thromboembolic events after left ventricular assist device implantation. Ann Thorac Surg 2016; 102:1543-1549. [PubMed: 27469338]

25. Efimova E, Fischer J, Bertagnolli L, et al. Predictors of ventricular arrhythmia after left ventricular assist device implantation: a large single-center observational study. Hear Rhythm 2017; 14:18121819.

26. Hickey KT, Colombo PC, Naka Y, et al. Atrial fibrillation is associated with recurrent ventricular arrhythmias after LVAD implant: Incidence and impact in a consecutive series. J Cardiovasc Transl Res 2019; 13:1-5.

27. Stulak JM, Deo S, Schirger J, et al. Preoperative atrial fibrillation increases risk of thromboembolic events after left ventricular assist device implantation. Ann Thorac Surg 2013; 96:2161-2167. [PubMed: 24035302]

28. Uriel N, Colombo PC, Cleveland JC, et al. Hemocompatibility-related outcomes in the MOMENTUM 3 trial at 6 months. Circulation 2017; 135:2003-2012. [PubMed: 28385948]

29. Hunter RJ, Berriman TJ, Diab I, et al. A randomized controlled trial of catheter ablation versus medical treatment of atrial fibrillation in heart failure (The CAMTAF Trial). Circ Arrhythmia Electrophysiol 2014; 7:31-38.

30. Di Biase L, Mohanty P, Mohanty S, et al. Ablation versus amiodarone for treatment ofpersistent atrial fibrillation in patients with congestive heart failure and an implanted device. Circulation 2016; 133:1637-1644. [PubMed: 27029350]

31. Marrouche NF, Brachmann J, Andresen D, et al. Catheter ablation for atrial fibrillation with heart failure. N Engl J Med 2018; 378:417-427. [PubMed: 29385358]

32. Maury P, Delmas C, Trouillet C, et al. First experience of percutaneous radio-frequency ablation for atrial flutter and atrial fibrillation in a patient with HeartMate II left ventricular assist device. J Interv Card Electrophysiol 2010; 29:63-67. [PubMed: 20393793]

33. Nguyen Thanh HK, Andrade J, Dyrda K, et al. MediGuide-assisted atrial flutter ablation in a patient with a HeartMate II left ventricular assist device. Hear Case Rep 2015; 1:290-292.

34. Vukelic S, Vlismas PP, Patel SR, et al. Digoxin Is associated with a decreased incidence of angiodysplasia-related gastrointestinal bleeding in patients with continuous-flow left ventricular assist devices. Circ Heart Fail 2018; 11:e004899. [PubMed: 30354557]

35. Ozcan C, Jahangir A, Friedman P, et al. Atrial fibrillation: long-term survival after ablation of the atrioventricular node and implantation of a permanent pacemaker in patients with atrial fibrillation. N Engl J Med 2001; 344:1043-1051. [PubMed: 11287974]

36. Mehra MR, Uriel N, Naka Y, et al. A full magnetically levitated left ventricular assist device-Final report. N Engl J Med 2019; 380:1618-1627. [PubMed: 30883052] 


\section{KEY POINTS}

- Atrial arrhythmias, particularly atrial fibrillation, are highly prevalent in patients with LVADs.

- Atrial arrhythmias are important determining factor of overall clinical outcome in LVAD patients.

- $\quad$ Rhythm versus rate control strategies in management of atrial arrhythmias in LVAD patients provide similar outcome.

- Anticoagulation and cardiovascular implantable electronic devices should be closely monitored and optimized in LVAD patients with atrial arrhythmias.

- $\quad$ Further studies are warranted to determine optimal management strategies and prevention of atrial arrhythmias in LVAD population. 


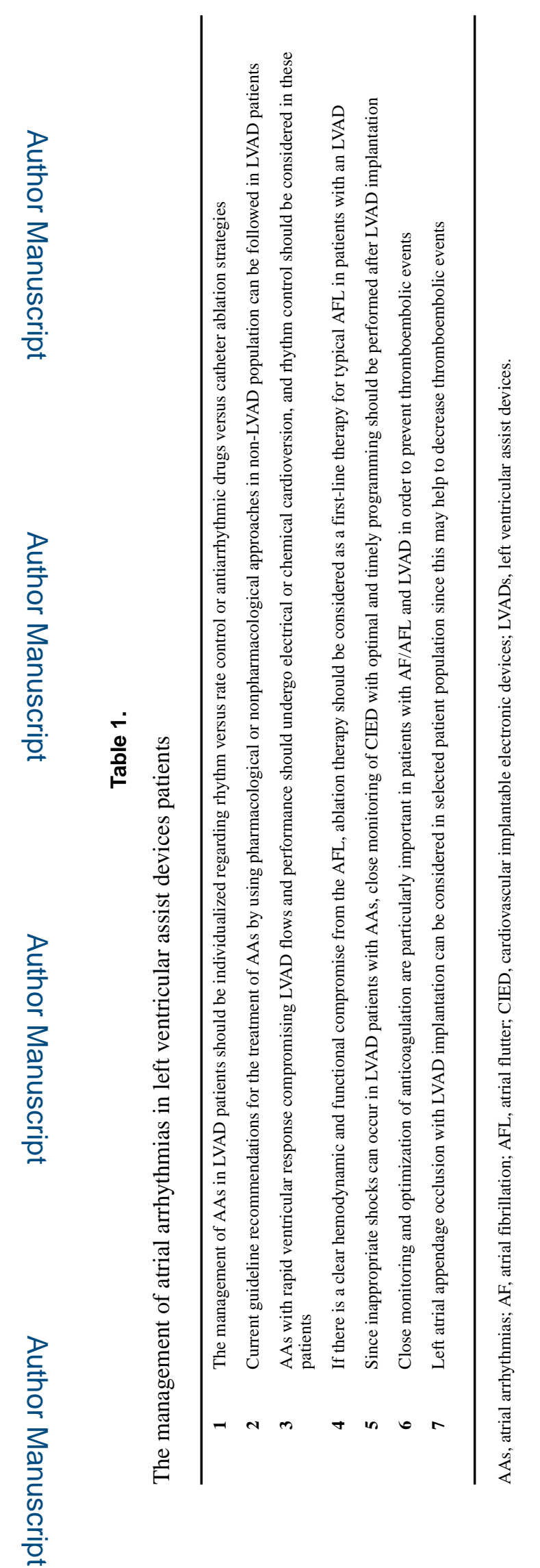

Curr Opin Cardiol. Author manuscript; available in PMC 2020 May 01. 